Ann. Biol. anim. Bioch. Biophys., I972, 12 (I), 5-II.

\title{
ÉVALUATION DES RESERVES SPERMATIQUES DANS LES VOIES DÉFÉRENTES DU COQ
}

\author{
Michel de REVIERS \\ avec la collaboration technique de J.-P. BRILLARD \\ Station de Recherches avicoles, \\ Centre de Recherches de Tours, I. N.R. A., \\ 37 - Nouzilly \\ RÉSUMÉ
}

20 coqs adultes ont été répartis en 2 lots : les uns (12 coqs) n'ont subi aucun prélèvement de sperme ; les autres (8 coqs) ont été prélevés quotidiennement pendant $25 \mathrm{j}$. Nous avons déterminé chez ces animaux :

- le nombre testiculaire ou nombre total de spermatides allongées et de spermatozoïdes par testicule ;

- les réserves spermatiques des voies déférentes.

Nous avons obtenu les résultats suivants :

Io Les réserves spermatiques des voies déférentes sont principalement contenues dans la moitié distale des canaux déférents qui ont chez le Coq un rôle d'organe de réserve analogue à celui de l'épididyme des Mammifères.

$2^{\circ}$ Il existe une corrélation élevée entre le nombre total $R_{t}$ de spermatozoïdes contenus dans les voies déférentes et les nombres testiculaires $\mathrm{Nt}(r=0,60$ chez les coqs non prélevés et $0,7^{8} \mathrm{chez}$ les coqs prélevés quotidiennement).

$3^{\circ}$ Le rapport $R_{t} / N_{t}$ est de $I, 65$ chez les coqs non prélevés et de 0,44 chez les coqs prélevés quotidiennement. En effet les collectes successives de sperme provoquent une déplétion des réserves spermatiques. Cette déplétion n'affecte que la moitié distale des canaux déférents.

\section{INTRODUCTION}

L'épididyme des Mammifères contient d'importantes réserves spermatiques (cf. revue d'AMANN, I970) dont il faut tenir compte pour mesurer la production spermatique réelle au niveau des éjaculats.

Chez le Coq, plusieurs auteurs ont signalé l'existence de réserves de sperme dans les canaux déférents du Coq (notamment LAKE et EI, JACK; I964) ; par contre, 
l'épididyme ne contiendrait qu'un petit nombre de spermatozoïdes dans cette espèce.

L'étude quantitative des réserves spermatiques des voies déférentes du Coq n'a pas été faite. Nous l'avons entreprise afin de déterminer leur influence éventuelle sur le nombre de spermatozoïdes collectés dans les éjaculats.

\section{MATÉRIEL E'T MÉTHODES}

Nous avons travaillé sur 20 coqs de même souche ( $M$ 55) de même âge (8-9 mois) et élevés dans les mêmes conditions. Ces coqs ont été maintenus en cages individuelles et soumis aux variations climatiques locales dans un abri ouvert, notre expérience s'étant déroulée en automne.

Les animaux ont été séparés au hasard en 2 lots :

- les uns (I2 coqs) n'ont subi aucun prélèvement de sperme ;

- les autres ( 8 coqs) ont subi chacun 25 prélèvements quotidiens de sperme, suivant la technique de Burrows et QuINN (1937).

Les coqs du lot non prélevé ont été abattus au cours de la période expérimentale et ceux du lot prélevé dans les 30 minutes après la dernière éjaculation.

Les testicules et les voies déférentes ont été prélevés et pesés individuellement aussitôt après l'abattage. Un fragment de 0,5 à I $\mathrm{g}$, pesé à I $\mathrm{mg}$ près et pris dans la région équatoriale de chaque testicule a été broyé dans du saccharose $0,25 \mathrm{M}$. Les voies déférentes ont été séparées en 4 segments suivant la figure $\mathrm{I}$ : "région épididymaire ", moitiés proximale et distale des canaux déférents, "vésicule séminale ". Ces échantillors ont été broyés de la même manière que pour les testicules, après mise au point de la technique de broyage (cf. Résultats, I).

Ces broyats ont été mis en suspensions homogènes dans du saccharose o, $25 \mathrm{M}$ q.s.p. roo ou $200 \mathrm{ml}$. Les spermatides allongées et les spermatozoïdes ont été dénombrés par comptages hématimétriques après ${ }_{5}$ minutes de décantation. Le nombre de spermatozoïdes par éjaculat a été déterminé de la même façon.

Les résultats de comptages hématimétriques sont assez variables d'une grille d'hématimètre à l'autre. Nous avons donc compté ro grilles par échantillon et en avons calculé la moyenne. Dans ces conditions, le coefficient de variation de la moyenne $\left(s_{m} / m\right)$ est de l'ordre de $3 \mathrm{p}$. 100 .

A la suite d'Ortavant (1958), nous appelons " Nombre testiculaire " le nombre total par testicule de spermatides allongées et de spermatozoïdes, et " coefficient d'activité testiculaire *, le rapport Nombre/Poids testiculaires.

\section{RÉSULTATS}

\section{I. - Influence des conditions de broyage sur l'évaluation du nombre testiculaire}

Appliquées pendant Io secondes, des vitesses de rotation élevées (Io 000 ou $20000 \mathrm{t} / \mathrm{mn}$ ) entraînent le bris de nombreuses têtes de spermatides et de spermatozoides qui ne peuvent alors être dénombrées.

Le tableau I montre qu'entre I ooo et Io ooo $\mathrm{t} / \mathrm{mn}$ la vitesse de rotation du broyeur et la durée de broyage ont une influence importante et très hautement significative sur les numérations; il $\mathrm{y}$ a, de plus, une interaction hautement significative entre ces deux traitements.

C'est la vitesse de $5000 \mathrm{t} / \mathrm{mn}$ qui donne les résultats les plus élevés : il est alors indifférent de broyer pendant 15 ou 30 secondes. Les phénomènes ont la même allure pour les voies déférentes mais n'apparaissent pas aussi nettement.

Ainsi préparés, les broyats d'organe peuvent être conservés I2 à $24 \mathrm{~h}$ à $+4^{\circ} \mathrm{C}$ sans changement significatif des nombres cellulaires. 


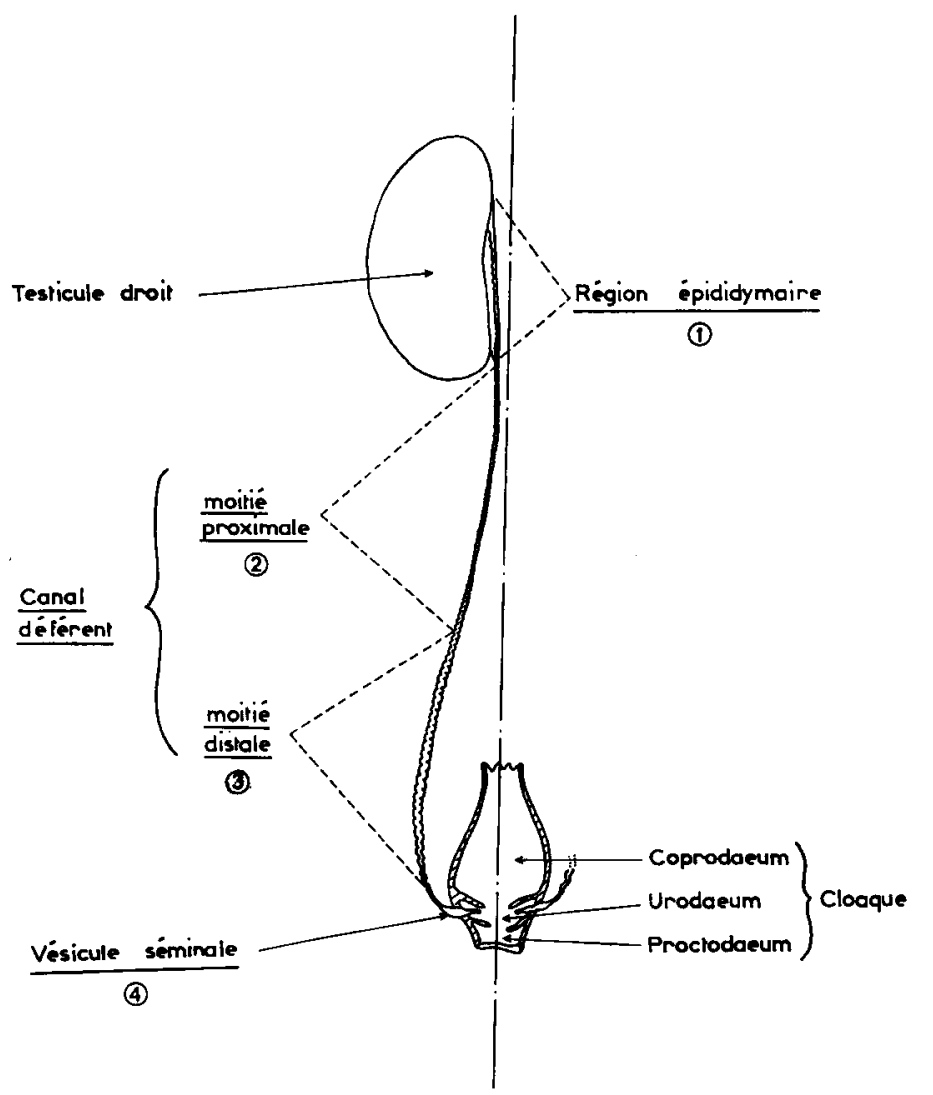

FIG. I. - Représentation schématique de l'appareil génital du Coq (I/2 vue droite) montrant l'échantillonnage effectué en vue de determiner les

\section{II. - Évaluation des réserves spermatiques des voies déférentes}

\section{Coqs non prélevés.}

Les réserves spermatiques totales des coqs non prélevés sont en moyenne de 6,8 milliards de spermatozoïdes (tab1. 2) ; 65 p. Ioo de ces réserves se trouvent dans la moitié distale des canaux déférents. Les réserves spermatiques totales varient beaucoup d'un coq à l'autre (o,88 à I9,0 milliards de spermatozoïdes) et cette variabilité provient surtout des fluctuations individuelles au niveau de la moitié distale des canaux déférents. Par ailleurs, les réserves spermatiques totales $\mathbf{R}_{t}$ sont en corrélation significative $(r=0,60 ; \mathrm{P}<0,05)$ avec les nombres testiculaires $\mathrm{N}_{t}$ (fig. 2 ). Ceci permet de définir le rapport $R_{t} / N_{t}$ qui est égal à $I, 65$ pour les coqs non prélevés (fig. 2).

2. Coqs prélevés quotidiennement.

Après 2 semaines de massages quotidiens d'entraînement, la récolte moyenne quotidienne de spermatozoïdes est d'environ I milliard par Coq avec de grandes fluctuations d'un animal à l'autre. 


\section{TABLEAU I}

Influence de la vitesse de rolation du broyeur et du temps de broyage sur le nombre de spermatides allongées comptées dans les broyats d'un même testicule de coq

(dilueur : Saccharose $0,25 \mathrm{M}$, ro répétitions par traitement résultats exprimés en millions de spermatides allongées par gramme de testicule)

\begin{tabular}{|c|c|c|c|}
\hline \multirow[b]{2}{*}{$\begin{array}{l}\text { Vitesse de rotation } \\
\qquad(\mathrm{t} / \mathrm{mI})\end{array}$} & \multicolumn{3}{|c|}{ Durée de broyage } \\
\hline & $\begin{array}{l}15 \text { secondes } \\
\left(m \pm s_{m}\right)\end{array}$ & $\begin{array}{c}30 \text { secondes } \\
\left(m \pm s_{m}\right)\end{array}$ & $\begin{array}{c}60 \text { secondes } \\
\left(m \pm s_{m}\right)\end{array}$ \\
\hline $\begin{array}{r}\text { Lente } \\
(1000)\end{array}$ & $395 \pm 19$ & $392 \pm 15$ & $307 \pm 7$ \\
\hline $\begin{array}{l}\text { Moyenne } \\
(5000)\end{array}$ & $443 \pm 12$ & $448 \pm 15$ & $409 \pm 12$ \\
\hline $\begin{array}{l}\text { Rapide } \\
(10000)\end{array}$ & $311 \pm 13$ & $295 \pm 11$ & $300 \pm 8$ \\
\hline
\end{tabular}

ANALYSE DE VARIANCE

\begin{tabular}{|c|c|c|c|c|}
\hline $\begin{array}{c}\text { Source } \\
\text { de variation }\end{array}$ & $\begin{array}{l}\text { Somme des } \\
\text { " carrés " (1) }\end{array}$ & $\begin{array}{l}\text { Degrés de } \\
\text { liberté }\end{array}$ & Carré moyen & $\mathbf{F}$ \\
\hline Vitesse & 259680 & 2 & 129840 & $81 ; 9 * *$ \\
\hline Durée & 35514 & 2 & 17757 & $11,2 * *$ \\
\hline Interaction & 24749 & 4 & 6187 & $3,9 *$ \\
\hline Erreur & 128297 & 81 & 1584 & $\rightarrow$ \\
\hline Totaux & 448240 & 90 & & \\
\hline
\end{tabular}

signicatif au seuil $1 \%$

(1) Carrés des écarts à la moyenne

TABLEAU 2

Comparaison des réserves spermatiques des voies déférentes chez les coqs ayant subi ou non des prélèvements quotidiens de sperme

Les nombres entre parenthèses sont les extrêmes.

\begin{tabular}{|c|c|c|c|c|c|c|}
\hline \multirow{3}{*}{$\begin{array}{l}\text { Prélèvement } \\
\text { de } \\
\text { sperme }\end{array}$} & \multirow{3}{*}{$\begin{array}{c}\text { Nbre } \\
\text { de } \\
\text { Cogs }\end{array}$} & \multicolumn{5}{|c|}{ Réserves spermatiques $\left(m \pm s_{m}\right)$ en milliards de spermatozoïdes } \\
\hline & & \multirow{2}{*}{$\begin{array}{c}\text { Région } \\
\text { épididymaire }\end{array}$} & \multicolumn{2}{|c|}{ Canaux déférents } & \multirow{2}{*}{$\begin{array}{l}\text { Vésicules } \\
\text { séminales }\end{array}$} & \multirow{2}{*}{ Total } \\
\hline & & & $\begin{array}{c}\text { moitié } \\
\text { proximale }\end{array}$ & $\begin{array}{l}\text { moitie } \\
\text { distale }\end{array}$ & & \\
\hline Non & 12 & $\begin{array}{c}0,36 \pm 0,07 \\
5 \% \\
(0,04-0,74)\end{array}$ & $\begin{array}{c}1,1 \pm 0,19 \\
16 \% \\
(0,21-2,10)\end{array}$ & $\begin{array}{c}4,4 \pm 1,2 \\
65 \% \\
(0,58-16,3)\end{array}$ & $\begin{array}{c}1,0 \pm 0,19 \\
14 \% \\
(0,32-1,97)\end{array}$ & $\begin{array}{c}6,8 \pm 1,3 \\
(0,88-19,0)\end{array}$ \\
\hline Oui & 8 & $\begin{array}{c}0,31 \pm 0,08 \\
8 \% \\
(0,03-0,66)\end{array}$ & $\begin{array}{c}1,1 \pm 0,29 \\
30 \% \\
(0,03-0,66)\end{array}$ & $\begin{array}{c}1,7 \pm 0,40 \\
48 \% \\
(0,43-2,50)\end{array}$ & $\begin{array}{c}0,51 \pm 0,23 \\
14 \% \\
(0,07-2,10)\end{array}$ & $\begin{array}{c}3,7 \pm 0,9 \\
(0,60-7,00)\end{array}$ \\
\hline
\end{tabular}




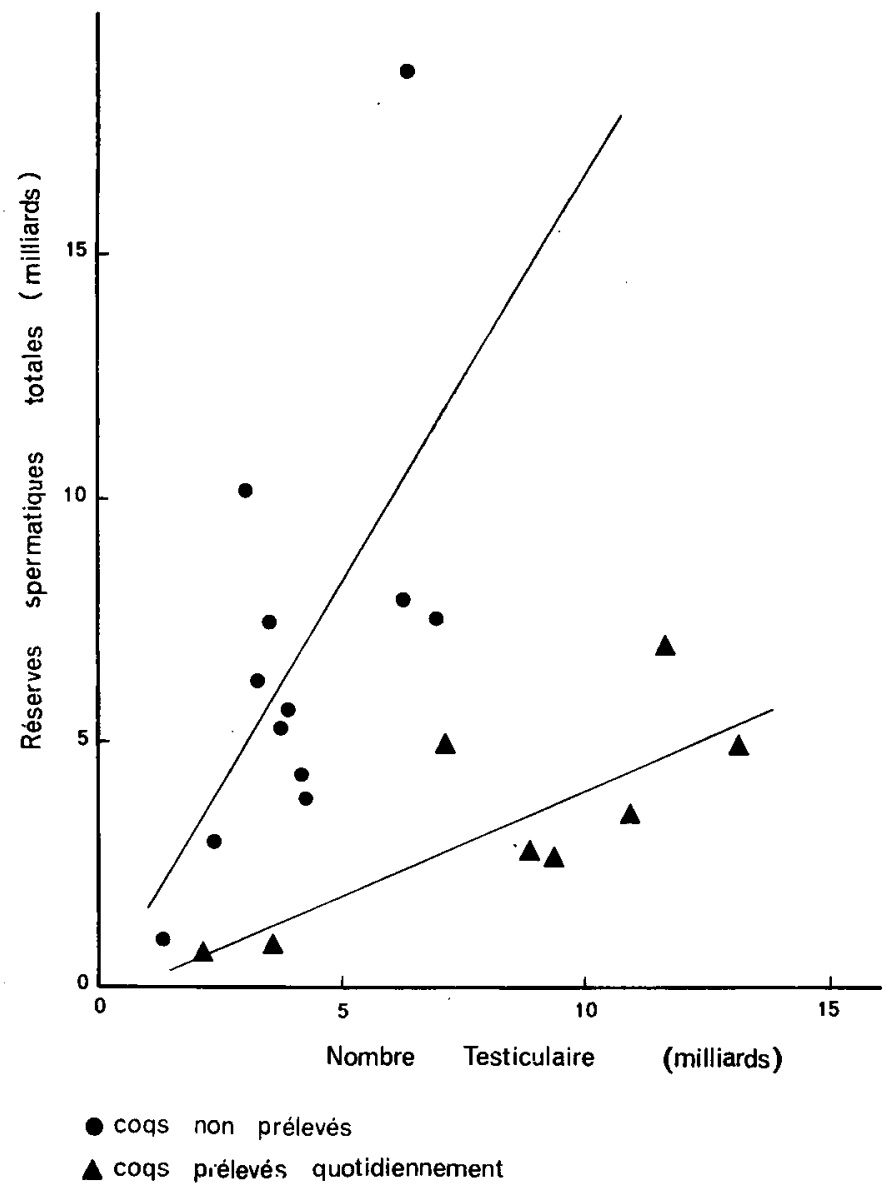

Fig. 2. - Relations entre les reserves spermatiques des voies deferentes $(\mathrm{Y})$ et les nombres testiculaires (X) chez des cogs ayant subi ou non des prélèvements quotidiens de sperme.

- Cogs non prélevés : $r=0,60 a \quad Y=1,65 X^{b}-0,02$

- Cogs prélevés : $\quad r=0,78$ a $\quad \mathrm{Y}=0,44 \mathrm{X}^{b}-0,30$

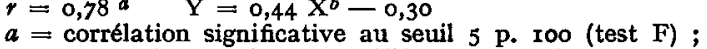

$b=$ pente significativement différente de o (test. $t$ ).

En fin d'expérience, les réserves spermatiques de ces coqs sont en moyenne de 3,7 milliards de spermatozoïdes (tabl. 2), donc inférieures à celles des coqs non prélevés. Cette différence ne porte que sur le contenu de la moitié distale des canaux déférents qui est environ 2,5 fois plus faible chez les coqs prélevés quotidiennement que chez les coqs non prélevés (tab1. 2). Le contenu moyen des autres segments de voies déférentes demeure inchangé. Il y a encore une grande variabilité individuelle des résultats (tabi. 2). Comme dans le cas précédent, les réserves spermatiques totales $\mathbf{R}_{\boldsymbol{t}}$ sont en corrélation significative élevée avec les nombres testiculaires $N_{t}(r=0,78$; $P<0,05)$ (fig. 2). Le rapport $\mathrm{R}_{t} / \mathrm{N}_{t}$ est égal à 0,44 chez les coqs prélevés quotidiennement (fig. 2).

Les collectes de sperme provoquent donc une diminution des réserves spermatiques des voies déférentes du coq ; cette diminution affecte essentiellement la moitié distale des canaux déférents. 


\section{DISCUSSION}

La figure 2 montre que les nombres testiculaires du lot prélevé sont supérieurs à ceux du lot non prélevé. Cette différence provient à la fois de variations du poids testiculaire total (respectivement; $\mathrm{I} 8, \mathrm{I} \pm 2,2$ et $\mathrm{I} 4,2 \pm 2,5 \mathrm{~g}$ ) et du coefficient d'activité testiculaire (respectivement $422 \pm 31$ et $294 \pm 26$ millions par g. de testicules).

Rappelons que les coqs du lot non prélevé ont été abattus pendant la période expérimentale alors que ceux du lot prélevé ont été abattus à la fin de cette période. Dans le premier cas, nous avons constaté une augmentation progressive des nombres testiculaires au cours du temps ; cette augmentation nous semble pouvoir expliquer les différences de caractéristiques testiculaires observées entre les deux lots de coqs.

Nous avons vu que les réserves spermatiques des voies déférentes du Coq sont surtout contenues dans la moitié distale du canal déférent. Ceci confirme les observations non quantifiées d'autres auteurs (LORENZ, I959; LAKE et EL, JACK, I964).

Par ailleurs, la fécondance des spermatozoïdes du Coq, faible au niveau épididymaire, augmente progressivement de la partie proximale à la partie distale des canaux déférents par suite d'un processus de maturation (MuNRo, I938). Il en est de même chez le Dindon (SAEKI et BROWN, I962).

Au plan fonctionnel le canal déférent des Oiseaux se présente donc comme l'homologue de l'épididyme des Mammifères (cf. revue d'AManN, I970).

Chez les deux groupes de Vertébrés, les collectes de sperme provoquent une déplétion des réserves spermatiques des voies déférentes et cette déplétion affecte surtout la partie distale des organes de réserve.

Puisque les collectes successives de sperme provoquent une déplétion des réserves spermatiques, on peut penser qu'elles ont aussi une influence sur la durée de séjour des spermatozoïdes dans les voies déférentes. En effet, Munro (I938) estime que la durée de transit des spermatozoïdes dans les voies déférentes est de 24 heures environ chez des coqs prélevés quotidiennement, ce que nous avons pu confirmer par marquage isotopique (de REVIERS, I968) mais selon MUNRo cette durée atteindrait 3 à 4 jours lorsque les coqs sont prélevés peu souvent.

Les réserves spermatiques doivent être assez rapidement éliminées dans des éjaculats consécutifs et rapprochés. Ainsi, en pratiquant plusieurs collectes le même jour, SAMPSON et WARREN (I939) observent que la quantité de sperme qui est émise à chaque éjaculat décroît rapidement et devient ensuite stable. PETITJEAN (I965) a pu confirmer cette observation en notant, de plus, que le temps au bout duquel cette stabilisation est acquise varie d'un coq à 1'autre. Cette dernière observation nous paraît provenir de l'importante variabilité individuelle des réserves spermatiques des voies déférentes.

En conclusion, on ne peut estimer la production spermatique des coqs à partir des éjaculats sans prendre un certain nombre de précautions. En effet, le nombre de spermatozoïdes contenus dans un éjaculat résulte à la fois de la production testiculaire et de l'état des réserves spermatiques. Pour éviter l'influence de ces réserves, il faut soit en établir le bilan avant et après l'expérience (ORTAVANT, I958), soit 
annuler ce bilan en effectuant un nombre suffisant de collectes avant de passer aux mesures, soit encore, épuiser les réserves par des collectes successives et très fréquentes juste avant la série de mesures.

Reçu pour publication en mai 1971.

\section{SUMMARY}

\section{ESTIMATION OF SEMEN CONTENTS}

OF THE VAS DEFERENS OF THE FOWL

20 adult roosters were investigated for " testis number * (i.e. total number of elongated spermatids and spermatozoa per testis) and sperm contents of the vas deferens. One batch of 8 birds was daily ejaculated for 25 days. Another batch of 12 birds was not ejaculated. Our results were as follows :

I. Vas deferens sperm was found to be stored mainly in the distal part of the ducts which act as storage sites in the fowl in the same way as the epididymis in Mammals.

2. There was a high correlation between total number of spermatozoa in the vas deferens (Rt) and testis number $(\mathrm{Nt}): r=0.60$ in non-ejaculated $v s .0 .78$ in ejaculated birds.

3. The Rt/Nt ratio was 1.65 in non-ejaculated $v s$. 0.44 in ejaculated birds, due to the fact that frequent collections induce a decrease in the amount of semen stored in the distal half of the vas deferens.

\section{RÉFÉRENCES BIBLIOGRAPHIQUES}

Amann R. P., 1970. Sperm production rates. in : The Testis, Johnson A. D., Gowes W. R., VAN Demark M. L., 1, 433-482.

BEDFORD J. M., I966. Development of the fertilizing abjlity of spermatozoa in the epididymis of the rabbit. J. Exp. Zool., 163, 319-329.

Blandau R. J., RUmery R. E., 1964. The relationship of swimming movements of epididymal spermatozoa to their fertilizing capacity. Fertil. Steril., 15, 57I-579.

Burrows W. H., Quinn J. P., 1935. A method of obtaining spermatozoa from the domestic fowl. Poult. Sci., 14, 25I-254.

Edwards J., I940. The effect of unilateral castration on spermatogenesis. Proc. Roy. Soc. London, B., 128, 407-42I.

LAKE P. E., 1957. The male reproductive tract of the fowl. J. Anat., 01, II6-128.

LAKE P. E., EL JACK M. H., r964. Further observations on the chemical composition of the seminal plasma of the domestic fowl. Ve Cong. intern. Repr. anim. Fec. Artif., Trento, 6-13 sept. 1964, 111, $359-363$.

Lorenz F. W., r959. Reproduction in the domestic fowl : Physiology of the male, in : Reproduction in domestic animals, Cole H. H., Cupps P. T., Acad. Press, New York, London, 11, 344-398.

Munro S. S., $193^{8}$ a. The effect of testis hormone on the preservation of sperm life in the vas deferens of the fowl. J. Exp. Biol., 15, I86-г96.

Munro S. S., I938 b. Functional changes in fowl sperm during their passage through the excurrent ducts of the male. J. Exp. Zool., 79, 71-92.

ORGEBIN-CRIST M. C., I 968 . Gonadal and epididymal sperm reserves in the rabbit : estimation of the daily sperm production. J. Reprod. Fert., 16, I5-25.

Ortavant R., I958. Le cycle spermatogenétique chez le Bélier. Thèse Doctorat ès Sciences, Paris, $127 \mathrm{pp}$.

SAMPSON F. R., WARREN D. C., 1939. Density of suspension and morphology of sperm in relation to fertility in the fowl. Poult. Sci., 18, 301-307.

Petitjean M., I965. Recherches sur l'estimation $d u$ pouvoir fécondant des Coqs. Thèse Ingénieur C. N. A. M., 79 p.

Reviers, M. de, I968. Détermination de la durée des processus spermatogénétiques chez le Coq à l'aide de thymidine tritiée. VI ${ }^{\circ}$ Cong. intern. Reprod. anim. Insem. artif., Paris, 1, 183-184.

Reviers M. de, I97I. Développement des testicules chez le Coq. II. Morphologie de l'épithélium séminifere et établissement de la spermatogenèse. Ann. Biol. anim. Biophys. Bioch. (sous presse.)

SACKI Y., BROWN K. I., I962. Fertilizing ability and laboratory evaluation of ejaculated and vasa deferentia semen in turkeys. Poult. Sci., 41, 905-909. 